\title{
Apparent Clearance for Unbound Drug
}

National Cancer Institute

\section{Source}

National Cancer Institute. Apparent Clearance for Unbound Drug. NCI Thesaurus. Code C154844.

The total apparent clearance of the unbound fraction of drug, adjusted for bioavailability. 